\title{
hQChain:
}

\section{Leveraging Towards Blockchain and Queueing Model for Secure Smart Connected Health}

\author{
Pratyusa Mukherjee, KIIT University (Deemed), India \\ LalBihari Barik, King Abdul Aziz University, Saudi Arabia \\ iD https://orcid.org/0000-0002-5977-6319 \\ Chittaranjan Pradhan, KIIT University (Deemed), India \\ (iD https://orcid.org/0000-0003-4928-7164 \\ Sudhansu Shekhar Patra, KIIT University (Deemed), India \\ (iD) https://orcid.org/0000-0001-9996-7681 \\ Rabindra K. Barik, KIIT University (Deemed), India \\ iD https://orcid.org/0000-0003-3086-3782
}

\begin{abstract}
Blockchain facilitates a broad spectrum of applications such as transaction of cryptocurrency, catering to financial services, designing, and constructing smart cities and so on. It has astounding benefits including accountability, consistency, and decentralization. Smart healthcare can be exemplified as utilizing propitious electronic technology safeguarded with blockchain for superior diagnosis of the disorders, improvised and cost-effective treatment of the patients, and enhanced quality of lives. Since blockchain in smart healthcare architecture hosts substantial amount of patient data, queueing models play a pivotal role to efficiently process the data. This paper highlights the concepts of blockchain, then delves into the smart healthcare architecture, and then deals with the several queueing models that already exist. It proposes the model (i.e., hQChain), which is inculcating $\mathrm{M} 1, \mathrm{~b} / \mathrm{Mb} / 1$ queueing model into blockchain-based smart healthcare architecture. It offers a queuing mathematical and analytical model to analyze and study the performance measurement of hQChain model.
\end{abstract}

\section{KEYWORDS}

Blockchain, Distributed Ledger, Electronic Health Record, Electronic Medical Record, Queueing Models, Smart Health, Smart Healthcare

\section{INTRODUCTION}

Blockchain provides an anonymous, accountable, authentic, consistent, decentralized and persistent approach for consensus impelled interaction amidst numerous entities through its distributed ledger (Carlozo, 2017). It has already revolutionized people's living standards by its immense impact on financial sectors, agriculture, transportation, healthcare and so on. Blockchain first came into limelight since the inception of Bitcoin by Santoshi Nakamoto (Nakamoto, 2008). It is essentially tampered proof as data is recorded in form of blocks and each block holds the corresponding data as well as 
Figure 1. Block diagram of a Blockchain

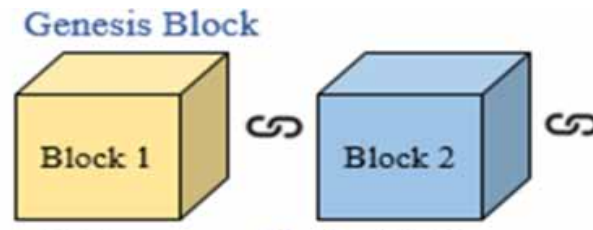

Hash : 3ZB4

Hash : 8B6Z

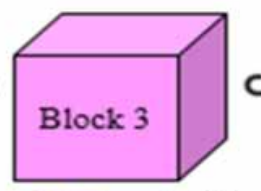

Hash : 765B

Previous Hash: 0000 Previous Hash: 3ZB4 Previous Hash: 8B6Z

Figure 2. Google Trends Smart City and Smart Health Comparison
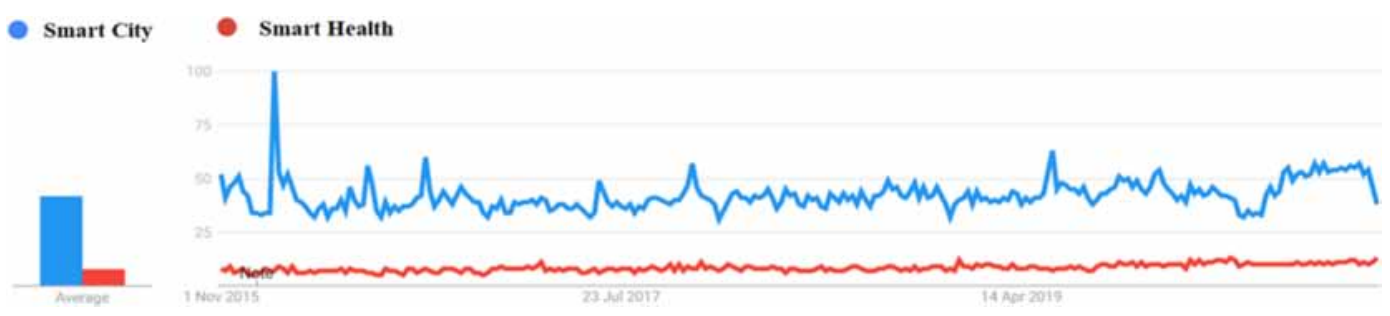

the cryptographic hash of its preceding block. Figure 1 illustrates the same. If any unauthorised amendment is made on any block, its hash immediately changes and hence there is a hash mismatch with its successor and the tampered block can be easily recognized.

Blockchain is widely used to enhance the security in several components of a smart city. Smart city is a signification bestowed to a city that ingests current information and communication technologies (ICT) to boost the quality of life for its dwellers (Deakin and Waer, 2011). Healthcare is treated as the preeminent fundamental facility and a city where hale and hearty citizens inhabit is equitable in every scenario. As a result of this, over the years, several researchers are inclined to work in the domain of smart health. Figure 2 gives the worldwide last five years comparison of Google Trends interest in Smart City and Smart Health. Due to its fundamental features, Blockchain based Healthcare is also garnering interest of researchers as illustrated in Figure 3.

Figure 4 represents the major healthcare goals:

- Disease Prevention: Disease prevention is a practice by which patients, specifically those with huge risk factors, are continually supervised and evaluated to foil the ailment from arising. Thus, the cure kickstarts even before symptoms of the disease, or shortly thereafter. To accomplish this

Figure 3. Google Trends Smart Health and Blockchain in Healthcare Comparison

- Smart Health Blockchain in Healthcare
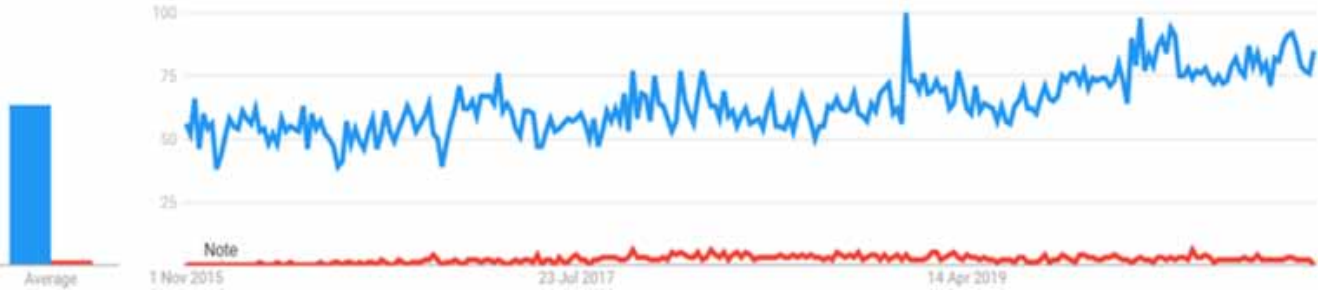


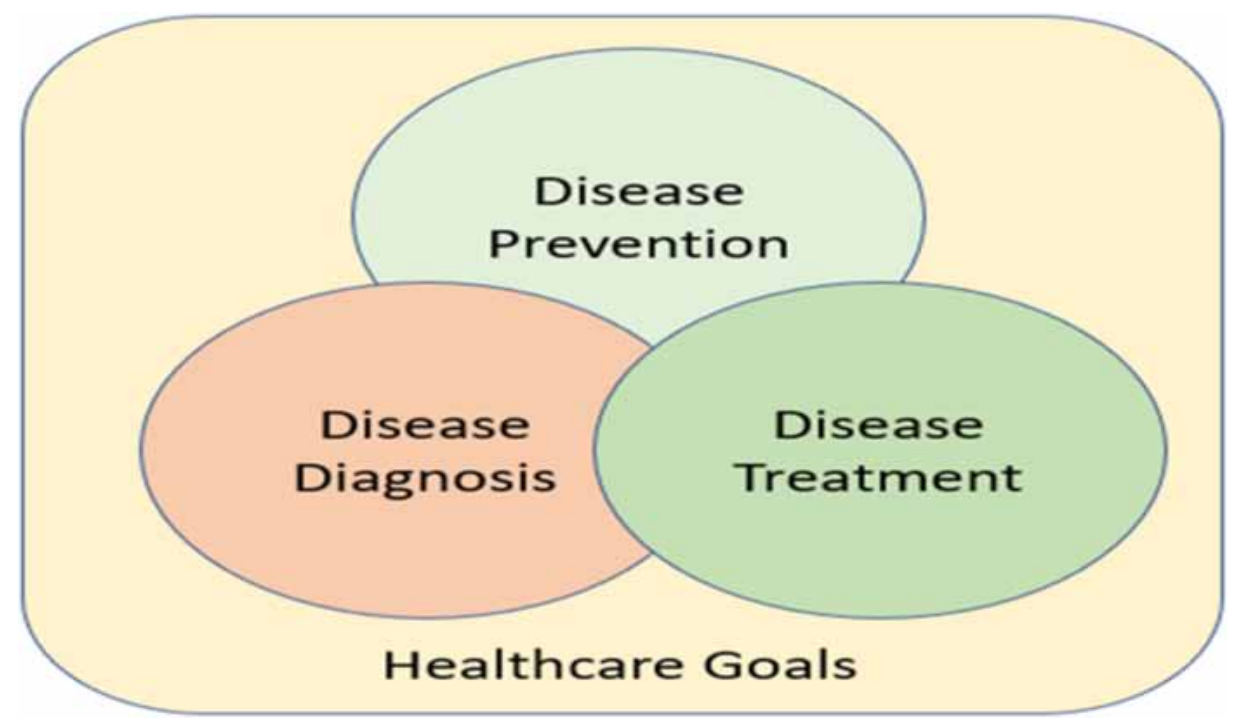

possible, the patient has to be monitored regularly. It thus requires persistent fitness check-ups, activity surveillance and analysis, disease prognosis etc.

- Disease Diagnosis: After patient shows certain symptoms of disorder, these need to be properly diagnosed to provide the best treatment. Diagnosis involves initial and detailed assessment by studying the patient history, evaluation of his major complaints and symptoms, prescribing certain medical tests. After the tests are conducted, the performance of the patient, interpretation of the outcomes is vital.

- Disease Treatment: Physician or specialist check-ups, follow-ups, conduct of medical procedures like surgery etc, patient being admitted to the hospital and his successive discharge and further consultations, proper medicines being prescribed play an important agenda in the course of the entire treatment.

All these three thus are essentially important to assure the best of the health of the citizens.

With the ever-increasing population, it becomes extensively critical to ensure wellbeing of the citizens in an instantaneous and efficient manner. It calls for innovation of smart devices and techniques which constantly monitor one's health and also administer timely solutions and treatment. Smart healthcare reposits the data conveyed by the smart health devices and this is termed as Electronic Medical Record (EMR) (Hillestad et al., 2005). This data is then inspected by health care professionals for improved diagnosis and treatment. This digital record keeping, and analysis are both cost and time effective for both patients and hospitals (Kemkarl and Dahikar,2012). Blockchain has the potential to provide doctors, medical practitioners as well as patients a guarded and regulated transfer of sensitive data. Thus, it can revamp data sharing and promoted transparency between clinical and systems. It also illustrated a proposed model of how Blockchain administers a significant platform for healthcare organizations to serve more effective treatment and diagnosis through potentially safe and secure data sharing even from remote ends. A patient notices symptom and seeks cure. Any healthcare operator can access his medical history from the blockchain and gather knowledge about his current clinical status. In this same blockchain platform, doctors can order patients diagnostic tests, perform them as well as interpret their outcomes (Pratyusa Mukherjee et al., 2021). In near future, similar tests can be avoided by the patient and hence its more economic for him. An array of professionals can proffer 
the best treatment to the patient and administer post follow ups and make sure the patient adheres to all measures to stay fit and healthy. Optimal functioning of blockchain calls for adequate processing of this huge amount with minimal waiting time. Thus, this calls for the formulation of blockchain queues. A queueing model (Obulor and Eke, 2016) needs to be constructed so that queue lengths and waiting time can be predicted and analytical decisions can be taken.

The prime emphasis of this paper is enlisted below:

- Study the incorporation of blockchain in smart healthcare.

- Highlight the concept of queueing in blockchain.

- $\quad$ Propose $h Q C h a i n$ model to a working model to adjoin queueing models in blockchain.

- Demonstrates the performance measures and numerical results.

Section 1 gives an elaborate introduction to the topic. The related work is discussed in detail in Section 2. It is divided in separate sub sections giving the nitty-gritty of existing smart healthcare techniques using Internet of Medical things, Big Data followed by application of Artificial Intelligence and Machine Learning in the field of healthcare. Section two also discusses the already available blockchain based Healthcare models and highlights the necessity to include queueing models for the same. Section 3 gives the proposed Smart Healthcare Architecture inculcated with Markovian Single Server. The performance measures are discussed in section 4 and numerical results are demonstrated in Section 5. Section 6 finally concludes our finding and throws some light on our future endeavours.

\section{RELATED WORK}

Continuous data sharing amidst patients and health care providers is an essential component of Smart Healthcare. mHealth and eHealth are two vital pillars of on which smart healthcare system rests. eHealth (Norman and Skinner,, 2006) or electronic health makes use of computers and internet to reposit and supervise medical records instead of paper files. mHealth (Kay et al., 2011) or mobile health performs medication and healthcare using mobile phones, computers or tabs. mHealth thus can be considered as a subset of eHealth.

\section{Existing Techniques for Smart Healthcare Using Internet of Things}

Internet of Medical Things (IOMT) facilitates identification, surveillance and intimidation to healthcare providers regarding the patient's vital stats at a very early stage to circumvent any severity in future (Joyia et al., 2017). Smart health devices can be utilized to track health statistics such as heart rates, blood glucose levels and sleeping patterns. Such devices can also be trained to remind them of day to day medications. Information collected from these devices by $24 * 7$ observation of the patients are analysed and integrated instantly. These are then immediately communicated to doctors in realtime which enhances the efficiency of healthcare system (Lee, 2011, Kumar and Venkatesan, 2014, Meyer et al., 2016).

Shima Okada et al. have developed a system which scrutinizes the body stats during sleep hours because the body nuances are predominantly correlated to the sleep-wake cycles (Okada et al., 2012). A remote IoT health care monitoring system was delineated by M. S. Kiran et al. that integrated the medical data from biomedical sensors and instantaneously translated it to the adjoining gateway for essential processing (Kiran et al., 2014). Iuliana Chiuchisan et al. framed the architecture for a health care system to outsee patients in smart Intensive Care Units (ICU) (Chiuchisan et al., 2014).

Luca Catarinucci et al. proffered a IoT based Smart Health System which enables automated tracking of patient, nursing staff and biomedical devices within the hospital environment as well as remote monitoring situations. They illustrated that their proposed architecture is equally capable to tackle emergencies immediately (Luca Catarinucci et al., 2015). Bhoomika. B.K and K N Muralidhara 
proposed a secured IoT based health monitoring system by using the PIC18F46K22 microcontroller as a doorway to communicate to several sensors designated to track the temperature, pulse rate, ECG or blood pressure of patients. Data collected by the microcontroller which is encrypted using standard AES128 is then sent it to the concerned doctors through a password protected network Wi-Fi module to present real time monitoring of the health (Bhoomika and Muralidhara, 2015). The controller is also connected with a buzzer to alert the caretaker about variation in sensor output thus enabling a quick provisional medication for the patient. It demonstrated an IoT supported healthcare kit which can be deployed as a mobile app to enable doctors to examine their patients from anywhere and everywhere and avert any emergency medical situations (Punit Gupta et al., 2016). Several other IoT based Smart Healthcare architectures are also suggested (Hongxu Yin et al., 2018 and Hongxu Zhu et al., 2019). Liyakathunisa Syed et al. put forward a novel smart healthcare big data framework to remotely monitor, compare and analyze physical daily activities of healthy and unhealthy population (L Syed et al.,2019). Another Smart Healthcare System in IoT environment can monitor a patient's rudimentary health conditions as well as the room condition where the patient is in real-time using five sensors in order to capture particular details using a heartbeat sensor, a body temperature sensor, a room temperature sensor, a CO sensor and a CO2 sensor (Md. Milon Islam et al., 2020).

Certain obstacles prevail in using IoT in Healthcare. Design issue is a predominant one. IoT based architectures are very robust and complex as all nearby devices must be able to interact with each other constantly (Solanas, et al., 2017; Baker et al., 2017). There is no definite standardization to design the most efficient IoT system that can bridge the gap between service providers and users. Privacy in Smart Healthcare plays a very crucial role as highlighted (He et al., 2018). The information assembled should be strictly confidential and safeguarded from any intrusion. All the smart devices must highly synchronised for proper functioning.

\section{Existing Techniques for Smart Healthcare Using Big Data}

Big Data in Healthcare refers to the abundant health data accumulated from diverse sources which is available in gigantic amount and is highly variable and diverse in structure and nature. Figure 5 mentions the several sources of big data in healthcare.

It proposed a cloud-based architecture to deal with plentiful amount of data requests from health service providers (He et al., 2012). It also recommended a distributed framework to store and examine huge quantities of approximately 77GB EEG data (Sahoo et al., 2013 and Jayapandian et al., 2013). Their proffered system Cloudwave processed five EEG reports in 1 minute, whereas the traditional system took more than 20 minutes. It also revealed that integrating Big Data into healthcare can provide an insight to several issues like how healthcare expenses vary geographically, which treatment is more prevalent in which disease, why are patients more inclined towards a particular health provider etc. (Sukumar et al., 2015). Zhe Yang et al. gave an IoT and Cloud amalgamated approach for real time collection and display of ECG data to in the preliminary diagnosis of certain heart disorders (Zhe Yang et al., 2016).

The incorporation of Bigdata into Healthcare also poses certain difficulties. The availability of several Bigdata technologies, their scalability, ease of use, ability to manipulate at different levels remain a serious issue. Quality of data stored and analysed, its confidentiality and privacy are also critical. There is a strong gap between data collection and processing (Zeadally et al., 2019). Bigdata technologies need to be menu driven, user friendly as well as more transparent for easy implementation. Healthcare data is highly fragmented thus demands constant data acquisition and cleansing to pave way for new data.

\section{Existing Techniques for Smart Healthcare Using Artificial Intelligence and Machine Learning}

Incorporation of Machine Learning in the healthcare systems has offered new dimensions in the smart healthcare. Google has lately started utilizing machine learning algorithms to detect cancerous 


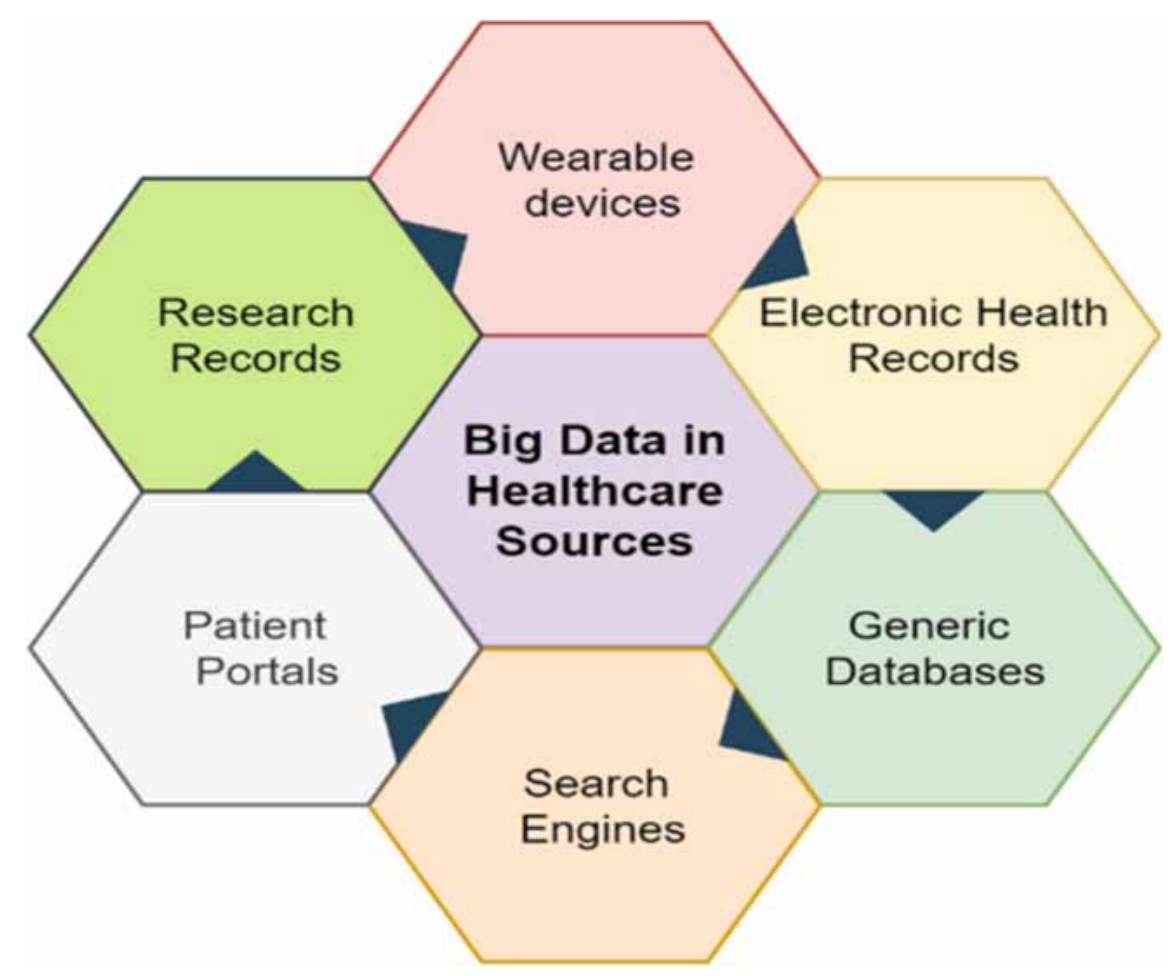

tumours on mammograms. Benefits of AI in Healthcare are plenty. It assists medical practitioners for better decision making to improve the accuracy of diagnosis. This better treatment thus reduces human error and chances of fatality. It has studied the prospects and contribution of machine learning in medical diagnosis. By providing online care and assistance through chatbots, AI reduces the patient's frequency to visit healthcare centres thereby optimizing the cost. Medication reminders can also be provided by AI an ML based healthcare (Kononenko et al., 2011). Harvard Medical School engages AI to help diagnose and treat patients more quickly as AI prove to be excellent and reliable symptoms checker. AI enabled machinery provides assistance to radiologists to automatically analyse the scans and reports for more assured diagnosis. BioXcel Therapeutics uses AI to recognize and develop new medicines in the domains of immuno-oncology and neuroscience, to enable drug re-innovation to discover new applications for existing drugs or to identify new patients.

It has analysed the utilization of Machine Learning in cancer forecasting and prognosis by analysing the cancer susceptibility, prediction of survivability and recurrence. Deep learning algorithm are being incorporated by Stanford University for investigating possibility of skin cancer (Joseph A. Cruz and David S. Wisharts, 2006). Andre It also observed that clinical images to identify skin cancer subtypes using neural networks. The procedure of drug creation can also be empowered by AI-powered programs which can reduce the expenses and overheads of developing pharmaceuticals through traditional clinical trials (Esteva et al., 2017).

Several challenges are also associated to healthcare with artificial intelligence or machine learning. A contrasting variation between training data and real-world data may lead to inappropriate conclusion which might result in wrong diagnosis and fatality. Machine learning applications usually function as a "black box" where decision-making is solely independent of human interference. Thus, there is no proper method to judge the accuracy of conclusions inferred by such applications. With time, the training datasets get outdated and contradict with the inevitable reality in changing medical practices, 
medications available and changes in disease characteristics and symptoms. Privacy regulations play a predominant role when it comes to sharing patient's experiences on a wider scale.

\section{Smart Healthcare Using Blockchain Technology}

Blockchain has is thus competent enough to have havoc applications in the domain of healthcare. It keeps an unsusceptible, decentralized, and transparent record of all patient data. The decentralized feature of this technology also enables every party to share the same information quickly and safely. This quick access reduces the diagnosis time and provides quicker treatment. Blockchains do not require any separate standardization and are highly scalable to be inculcated with other technologies. Blockchain also eases the medical supply chain and provides drug traceability. Several blockchain based Smart Healthcare Architectures have been proposed by many researchers in recent years.

Matthias Mettler discussed the potential application of Blockchain for healthcare regulation, medical research, and drug counterfeiting in the pharmaceutical sector (Mettler, 2016). It also presented their perspectives on blockchain based EMR data sharing between health-related service providers with full privacy, security and availability. This methodology reduces the turnaround time for health information sharing, quickens decision making and diminishes the overall cost (Alevtina Dubovitskaya et al., 2017). AF. da Conceição et al. [36] presented how blockchain and smart contracts could enhance data access, management, and interoperability in the healthcare sector (da Conceição et al., 2018). It also presented the challenges and obstacles that must be resolved before the effective absorption of blockchain technology in healthcare systems (Tanesh Kumar et al., 2018). They have utilized the concept of smart contract which is key to define the pre-defined agreements amongst the numerous stakeholders involved. It developed a blockchain based model to ensure data security, provide data provenance and enable patients full control of their vital health records (Yan Zhuang et al., 2020).

\section{Necessity of Queueing Models in Blockchain Technology}

It has been observed that most important steps are to develop the basic theory related to a blockchain, for example, mathematical models such as Markov processes and queueing theory for mining management and consensus mechanism, performance analysis and optimization of blockchain systems play crucial roles and demand more attention. The concept of blockchain queueing theory was introduced by Kasahara and Kawahara (Kasahara and Kawahara, 2016; Kawase and Kasahara, 2017), who proposed that the processing time follows continuous probability distribution. To draw effective services from the blockchain the entire task is segregated into two major stages. First is to focus on the block generation and blockchain building process. Second is to effectively process these blocks to draw valuable information about a patient's health to provide effective treatment.

A mining processes is used to maintain and update a blockchain where many nodes or participants, termed as miners, compete to solve a very difficult puzzle-like problem (Kiayias et al., 2016; Quan-Lin, 2018). Initially the patient records are organized into a block, and then the block is included into the blockchain after the nonce is decided by means of a mining competition that is an algorithmic puzzle specialized for this block. Service time is defined as the record confirmation time which is the sum of the block generation and blockchain building times. A block is a list of patient health data, together with metadata that consists of the timestamp of the current block, the timestamp of the immediately preceding block, and a field called a nonce which is given by the mining winner. Li et al. focused on developing queueing theory for blockchain systems using a Markovian batch service queueing system for cryptocurrencies for easy block generation and blockchain creation. They also expressed three major performance measures namely average number of records in the queue, average number of records in a block, average processing time (Li et al., 2018; Li et al., 2019).

Julian Barreiro-Gomez et al the concept of cryptographic tokens in blockchain as well as the behaviour of the agents depending on the incentive, network security and network delay (BarreiroGomez and Tembine, 2019). Stefan Geissler et al. developed a discrete-time queueing model that allows the evaluation of key performance indicators of blockchain and distributed ledger systems. 
Figure 6. hQChain: Queueing Model for the Blockchain based Healthcare system where the generation process of the blocks and the process of building the blocks are considered as two stages where the batch service works

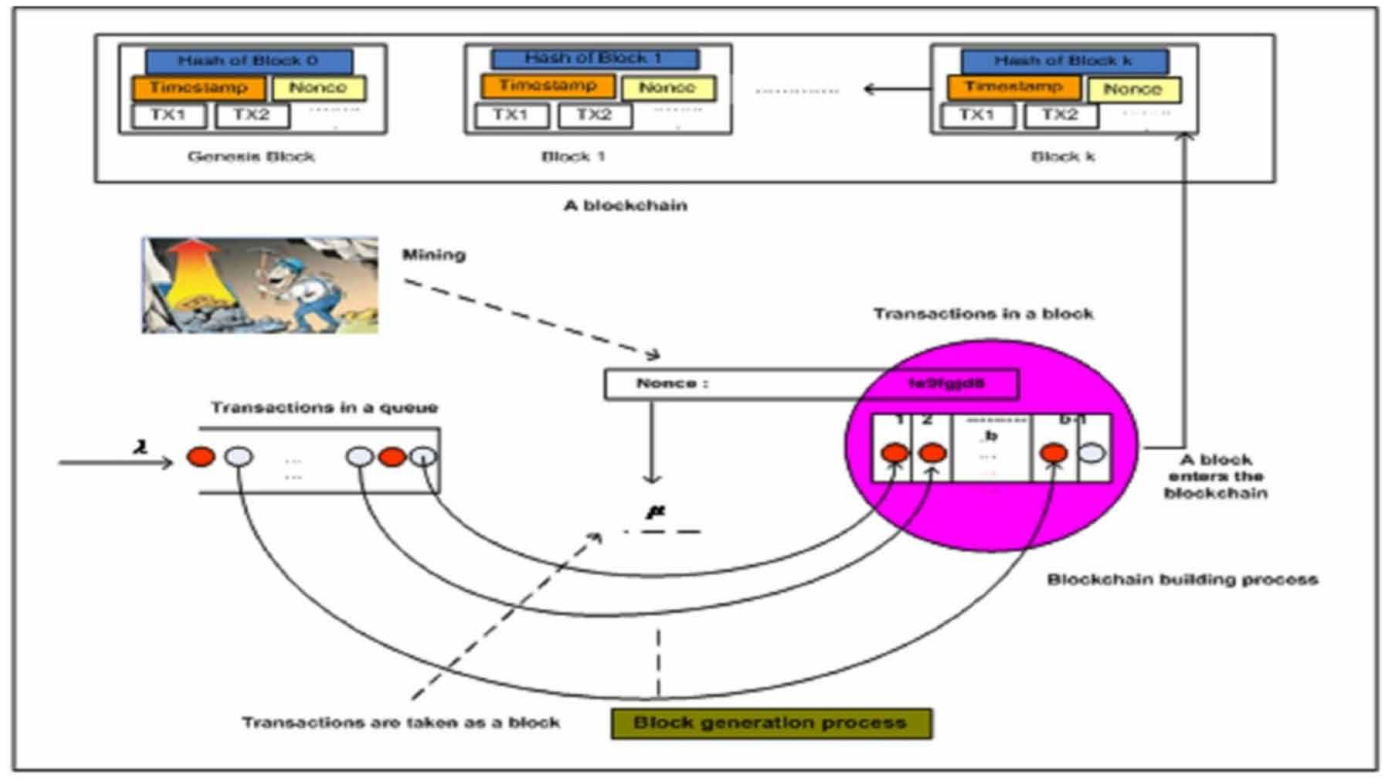

They analysed the queueing model based on the queue size and waiting time for each transaction (Geissler et al., 2019).

\section{PROPOSED MODEL}

It is thus evident that the blockchain is hosting tremendous amount of patient data ranging from his previous health records, already performed medical tests, procedures undergone, his regular medication information as well as vital stats. It is therefore very crucial to timely process as well as analyse this massive data to provide the best service. This feature calls for the incorporation of proper queueing models. Queueing model is required at three stages. Firstly, when health related data from several sources like the patient himself, doctors and health professionals is fed into the blockchain. Next withing the blockchain, efficient queueing is needed to process and analyse each data timely. Thirdly, from the blockchain when solution or treatment is to be sent to the patient's smartphone or medical professionals, it must be quick and appropriate.

Considering the functioning of the blockchain system, the system can be modelled as a blockchain queue which is termed as hQChain. Figure 6 illustrates the proposed hQChain model. Here the generation process of the blocks and the process of building the blocks are considered as two stages where the batch service works. During the block generation stage, the confirmation of a block is done with the help of a computational problem solving by a miner out of the miners using cryptographic hash algorithmic procedure, named mining. The nodes who contest to solve the problem are termed as miners. The miner who is the winner in the competition will be given some reward having some constant values as well as the charge of the transactions, and still has the authority for appending any newly arrival block into the system. A block is the collection of transactions, metadata which includes the timestamp of the newly added block, previous block, nonce given by the mining winner. In our model, the service time is considered as the sum of generation of blocks and blocks building process.

Some of the model descriptions of hQChain are defined as follows: 
- Arrival Process: The Transactions enters to the blockchain model with the Poisson process. The arrival rate to the system is defined as each transaction enters into the system, waits in the queue where there are infinite waiting room in the system. One transaction may join into the system or there may be $b$ number of transactions enter into the system. The lower left corner of fig (6) shows the arrival process of the transactions.

- Service Process: Every transaction once entered the system is queued up in a buffer that is infinite in size. During the first stage of service, known as the block creation process, the transactions are successfully mined into blocks. A set of transactions are taken in a block (let it be b), a mining winner added a nonce to the block. Finally, during the second stage of the service, the blockchain is pegged with a block having a group of transactions. The service rate is denoted by $\mu$ and is shown in the lower part of Figure 6.

- Discipline of Block-generation: A block is consisting of many transactions, but a maximum of $\mathrm{b}$ transactions can contain inside a block. The transactions inside the block do not necessarily follow FCFS w.r.t their arrivals. Some transactions those who arrived late at the queue may be mined into the block first. But for simplification, we have considered FCFS in our system model. The block generation process is depicted in the lower centre of Figure 6.

- Maximum block size: The maximum block size is limited, which helps in avoiding spam attacks. There are at most $b$ transactions that can be taken in a block. If there are more than $n$ transactions waiting in the waiting room, only at a maximum of $\mathrm{n}$ transactions are considered for the new block generation.

- Independence: Every random variable mentioned above will be independent of one another.

This paper models the system as a Markovian single server $\mathrm{M}^{1, \mathrm{~b}} / \mathrm{M}^{\mathrm{b}} / 1$ queueing system where the server serves the entire batch of transactions in the queue:

$\pi_{0}$ : The system has no transaction arrived at the queue for posting into the block.

$\pi_{b}$ : The system having $\mathrm{b}$ number of transactions arrived in the queue for posting into the block.

$\pi_{i}$ : The system having i number of transactions arrived in the queue for posting into the block $(0$ $<=\mathrm{i}<=\mathrm{b})$.

The balance equation for the system can be written as:

$$
\begin{aligned}
& (\lambda+2 \lambda+3 \lambda+\ldots .+b \lambda) \pi_{0}=\mu \pi_{b} \\
& =>\left(\lambda \frac{b(b+1)}{2}\right) \pi_{0}=\mu \pi_{b} \\
& =>\pi_{b}=\frac{\lambda}{\mu} \frac{b(b+1)}{2} \pi_{0} \\
& (\lambda+2 \lambda+3 \lambda+\ldots .+(b-i) \lambda) \pi_{i}=\lambda \pi_{i-1}+2 \lambda \pi_{i-2}+3 \lambda \pi_{i-3}+\ldots .+i \lambda \pi_{0}
\end{aligned}
$$

Let us define a relationship between $\pi_{1}$ and $\pi_{0}$ as: 
$\pi_{1}=q_{1} \pi_{0}$

From equations (1),(2) and (3), $\pi_{i}, 0<\mathrm{i}<\mathrm{b}$ can be defined in terms of $\pi_{0}$ as follows:

$\pi_{2}=q_{2}\left(q_{1}+2\right) \pi_{0}$

$\pi_{3}=q_{3}\left(q_{2}\left(q_{1}+2\right)+2 q_{1}+3\right) \pi_{0}$

$\pi_{4}=q_{4}\left(q_{3}\left(q_{2}\left(q_{1}+2\right)+2 q_{1}+3\right)+2 q_{2}\left(q_{1}+2\right)+3 q_{1}+4\right) \pi_{0}$

We know:

$\sum_{i=1}^{b} \pi_{i}=1$

$\pi_{i}$ can be generalized and expressed as:

$$
\pi_{i}=q_{i} \pi_{0}\left(\sum_{j=1}^{i} j\left(\sum_{k=1}^{i-j} k\left(\prod_{l=k}^{i-j} q_{l}\right)\right)+i\right)
$$

where $0<\mathrm{i}<\mathrm{b}$ :

$q_{i}=\frac{2}{(b-i)(b-i+1)}$

$\prod_{l=1}^{k-1} q_{l}=\prod_{l=1}^{k-1} \frac{2}{(b-l)(b-l+1)}=\frac{2^{k-1}}{\frac{(b !)^{2}}{(b)(b-k+1)}}=\frac{2^{k-1}(b)(b-k+1)}{(b !)^{2}}$

$=>\sum_{k=1}^{i-1}\left(\prod_{l=1}^{k-1} q_{l}\right) k=\sum_{k=1}^{i-1}\left(\frac{2^{k-1}(b)(b-k+1)}{(b !)^{2}}\right) k$ 
$\left.=\frac{b}{(b !)^{2}}\left((b+1)\left(\sum_{m=0}^{i-2} 2^{m} m\right)-\left(\sum_{m=0}^{i-2} 2^{m} m^{2}\right)+b \frac{2\left(2^{i-2}-1\right)}{(2-1)}\right)\right)$

The sigma functions used in equation (12) is expressed as follows:

$$
\begin{aligned}
& \sum_{m=0}^{i-2} 2^{m} m=2^{0} 0+2^{1} 1+2^{2} 2+\ldots .+2^{i-3}(i-3)+2^{i-2}(i-2) \\
& =2^{i-1}(i-3)+2 \\
& \sum_{m=0}^{i-2} 2^{m} m^{2}=2^{0} 0^{2}+2^{1} 1^{2}+2^{2} 2^{2}+\ldots .+2^{i-3}(i-3)^{2}+2^{i-2}(i-2)^{2} \\
& =2^{i-1}\left(i^{2}-6 i+11\right)-6
\end{aligned}
$$

Putting equations (13) and (14) in equation (12):

$$
\begin{aligned}
& \sum_{k=1}^{i-1}\left(\prod_{l=1}^{k-1} q_{l}\right) k=\frac{b}{(b !)^{2}}\left((b+1)\left(2^{i-1}(i-3)+2\right)+\left(b \frac{2\left(2^{i-2}-1\right)}{(2-1)}\right)+\right. \\
& \left.\left((-1)\left(2^{i-1}\left(i^{2}-6 i+11\right)-6\right)\right)\right) \\
& =>\sum_{j=1}^{i} j\left(\sum_{k=1}^{i-1}\left(\prod_{l=1}^{k-1} q_{l}\right) k\right)=\sum_{j=1}^{i} j\left[\frac { b } { ( b ! ) ^ { 2 } } \left((b+1)\left(2^{i-1}(i-3)+2\right)+\left(b \frac{2\left(2^{i-2}-1\right)}{(2-1)}\right)+\right.\right. \\
& \left.\left.\quad\left((-1)\left(2^{i-1}\left(i^{2}-6 i+11\right)-6\right)\right)\right)\right] \\
& =\sum_{j=1}^{i} j\left(\frac { b } { ( b ! ) ^ { 2 } } \left(\left(2^{i-1} i b-2^{i-1} 3 b+2 b+2^{i-1} i-2^{i-1} 3+2\right)\right.\right. \\
& \left.\left.\quad+\left(2^{i-1} b-2 b\right)+\left(-2^{(i-1)} i^{2}+2^{(i-1)} 6 i-2^{(i-1)} 11+6\right)\right)\right)
\end{aligned}
$$

The Stirling's Approximation is given by:

$$
b ! \sim \sqrt{2 \pi b}\left(\frac{b}{e}\right)^{b}
$$

Putting equation (18) in (17) and solving for $\pi \mathrm{i}$ :

$$
\grave{\mathrm{A}}_{1}=q_{i} \grave{\mathrm{A}}_{0}\left(\left(\frac{i(i+1)}{2}\right)\left(\frac{b}{\sqrt{2 \pi b}\left(\left(\frac{b}{e}\right)^{b}\right)^{2}}\right)\left(2^{i-1}\left(i b-2 b+7 i-14-i^{2}\right)+8\right)+i\right)
$$


where $0<\mathrm{i}<\mathrm{b}$ :

$$
q_{i}=\frac{2}{(b-i)(b-i+1)}
$$

Now equation (7) can be written as:

$$
\begin{aligned}
& \sum_{i=1}^{b} \pi_{i}=1 \\
& =>\pi_{0}+\sum_{i=1}^{b-1} \pi_{i}+\pi_{b}=1
\end{aligned}
$$

Solving:

$$
\pi_{0}=\frac{1}{\left(1+\sum_{i=1}^{b-1}\left(q_{i}\left(\sum_{j=1}^{i} j\left(\sum_{k=1}^{i-1}\left(\prod_{l=1}^{k-1} q_{l}\right) k\right)+i\right)\right)+\frac{\lambda}{\mu}\left(\frac{b(b+1)}{2}\right)\right)}
$$

where:

$$
\pi_{b}=\frac{\lambda}{\mu}\left(\frac{b(b+1)}{2}\right) \pi_{0}
$$

\section{PERFORMANCE MEASURES}

The following are some baseline performance measures of the proposed model:

Lq: The average number of transactions in the queue (i.e, the block currently mined):

$$
L_{q}=\sum_{i=0}^{b} i \pi_{i}
$$

where:

$$
\sum_{i=0}^{b} i \pi_{i}=\sum_{i=0}^{b} i\left(q_{i} \pi_{0}\left(\sum_{j=1}^{i} j\left(\sum_{k=1}^{i-1}\left(\prod_{l=1}^{k-1} q_{l}\right) k\right)+i\right)\right)
$$

2. Wq: The average amount of time a transaction in queue or the block being mined: 
Table 1. Mean waiting time of the transactions in the waiting Queue

\begin{tabular}{|l|l|l|l|l|l|l|l|l|l|}
\hline & \multicolumn{1}{|l}{$\lambda=0.05$} & \multicolumn{1}{l}{$\lambda=0.03$} & \multicolumn{2}{l|}{$\lambda=0.01$} \\
\hline $\mathrm{b}$ & $\mu=0.04$ & $\mu=0.06$ & $\mu=0.08$ & $\mu=0.04$ & $\mu=0.06$ & $\mu=0.08$ & $\mu=0.04$ & $\mu=0.06$ & $\mu=0.08$ \\
\hline 10 & 989.2623 & 781.1622 & 683.3933 & 894.5345 & 689.7567 & 668.1323 & 794.5959 & 696.1394 & 597.6455 \\
\hline 20 & 842.2272 & 682.7627 & 582.4303 & 783.4677 & 598.7567 & 564.1034 & 696.9549 & 597.4594 & 497.9404 \\
\hline 30 & 772.2278 & 528.1612 & 511.1344 & 697.7656 & 535.1345 & 467.8293 & 667.1939 & 497.9409 & 468.1324 \\
\hline 40 & 672.1729 & 489.7717 & 423.1424 & 683.1526 & 463.7263 & 357.6777 & 653.1526 & 468.7263 & 378.1454 \\
\hline 50 & 572.35278 & 428.1717 & 383.5524 & 593.3536 & 424.1737 & 346.1336 & 586.3536 & 446.1737 & 297.6645 \\
\hline 60 & 473.3627 & 329.6717 & 331.1322 & 483.3636 & 363.6723 & 235.8657 & 497.3636 & 397.6723 & 268.3256 \\
\hline
\end{tabular}

$W_{q}=\frac{L_{q}}{\lambda}$

3. W: The average amount of time a transaction in the system i.e., the transaction in the blockchain:

$$
W=W_{q}+\frac{1}{\mu}
$$

4. L: The average number of transactions in the system:

$$
L=\lambda W
$$

\section{NUMERICAL RESULTS}

For demonstrating the applicability of the above blockchain based queueing healthcare system, several numerical results have been carried out and few of them are presented here. Table 1 shows the average waiting time of the transactions in the waiting queue for different arrival rates $(\lambda)$ and different block sizes (b). The mean waiting time decreases in the queue when $b$ increases. It also increases when $\lambda$ decreases. Table 2 shows the variance of the waiting time in the queue for different arrival rate $(\lambda)$ and different block sizes (b). The variance decreases in the queue when $b$ increases. It also increases when $\lambda$ decreases.

The effect of queue length on mean system length(L). mean queue length (Lq), mean waiting time (W) of a transaction in the system, and in the buffer are presented in Figure 7-10 respectively for a fixed $b=5$.

Figure 11 illustrates dependence of blocking probability of the buffer size $\mathrm{N}$ varying from 1 to 30 and the batch size $b$ varying from 1 to 7 . The parameters are taken as $\lambda=0,7, \mu=52$. We observe that for fixed batch size the loss of probability decreases as the buffer size increases. Further with fixed buffer size it increases when the batch size increases. Hence, we can setup an admissible batch size and the sufficient buffer size in the system in order to have lower blocking probability. 
Table 2. Variance of waiting time of the transactions in the waiting Queue

\begin{tabular}{|c|l|l|l|l|l|l|l|l|l|}
\hline \multicolumn{3}{|l|}{$\lambda=0.05$} & \multicolumn{2}{l|}{$\lambda=0.03$} & \multicolumn{2}{l|}{$\lambda=0.01$} \\
\hline $\mathrm{b}$ & $\mu=0.04$ & $\mu=0.06$ & $\mu=0.08$ & $\mu=0.04$ & $\mu=0.06$ & $\mu=0.08$ & $\mu=0.04$ & $\mu=0.06$ & $\mu=0.08$ \\
\hline 10 & $\begin{array}{l}9.78 \\
\mathrm{E}+05\end{array}$ & $5.54 \mathrm{E}+05$ & $4.23 \mathrm{E}+05$ & $8.45 \mathrm{E}+05$ & $5.12 \mathrm{E}+05$ & $4.13 \mathrm{E}+05$ & $8.25 \mathrm{E}+05$ & $5.02 \mathrm{E}+05$ & $4.02 \mathrm{E}+05$ \\
\hline 20 & $\begin{array}{l}7.25 \\
\mathrm{E}+05\end{array}$ & $4.85 \mathrm{E}+05$ & $3.45 \mathrm{E}+05$ & $6.56 \mathrm{E}+05$ & $4.65 \mathrm{E}+05$ & $3.22 \mathrm{E}+05$ & $6.26 \mathrm{E}+05$ & $4.45 \mathrm{E}+05$ & $3.12 \mathrm{E}+05$ \\
\hline 30 & $\begin{array}{l}5.56 \mathrm{E} \\
+05\end{array}$ & $3.23 \mathrm{E}+05$ & $2.67 \mathrm{E}+05$ & $5.34 \mathrm{E}+05$ & $3.20 \mathrm{E}+05$ & $2.54 \mathrm{E}+05$ & $5.14 \mathrm{E}+05$ & $3.10 \mathrm{E}+05$ & $2.24 \mathrm{E}+05$ \\
\hline 40 & $\begin{array}{l}4.46 \\
\mathrm{E}+05\end{array}$ & $2.54 \mathrm{E}+05$ & $2.34 \mathrm{E}+05$ & $4.32 \mathrm{E}+05$ & $2.45 \mathrm{E}+05$ & $2.23 \mathrm{E}+05$ & $4.12 \mathrm{E}+05$ & $2.25 \mathrm{E}+05$ & $2.13 \mathrm{E}+05$ \\
\hline 50 & $\begin{array}{l}2.97 \\
\mathrm{E}+05\end{array}$ & $2.51 \mathrm{E}+05$ & $2.13 \mathrm{E}+05$ & $2.78 \mathrm{E}+05$ & $2.34 \mathrm{E}+05$ & $2.05 \mathrm{E}+05$ & $2.58 \mathrm{E}+05$ & $2.14 \mathrm{E}+05$ & $1.97 \mathrm{E}+05$ \\
\hline 60 & $\begin{array}{l}2.26 \\
\mathrm{E}+05\end{array}$ & $1.34 \mathrm{E}+05$ & $1.24 \mathrm{E}+05$ & $2.13 \mathrm{E}+05$ & $1.23 \mathrm{E}+05$ & $1.21 \mathrm{E}+05$ & $2.03 \mathrm{E}+05$ & $1.03 \mathrm{E}+05$ & $0.98 \mathrm{E}+05$ \\
\hline
\end{tabular}

Figure 7. Effect on $\mathrm{N}$ on $\mathrm{L}$

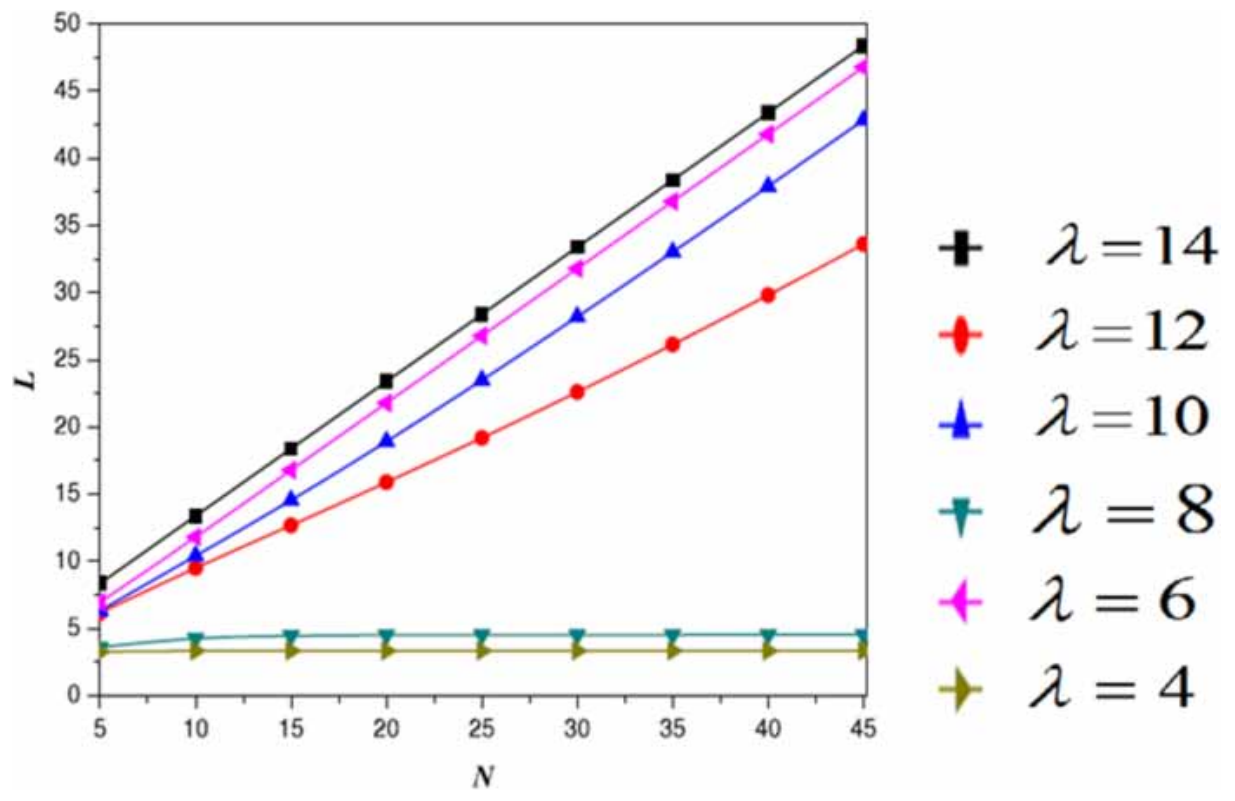


Figure 8. Effect of N Vs Lq

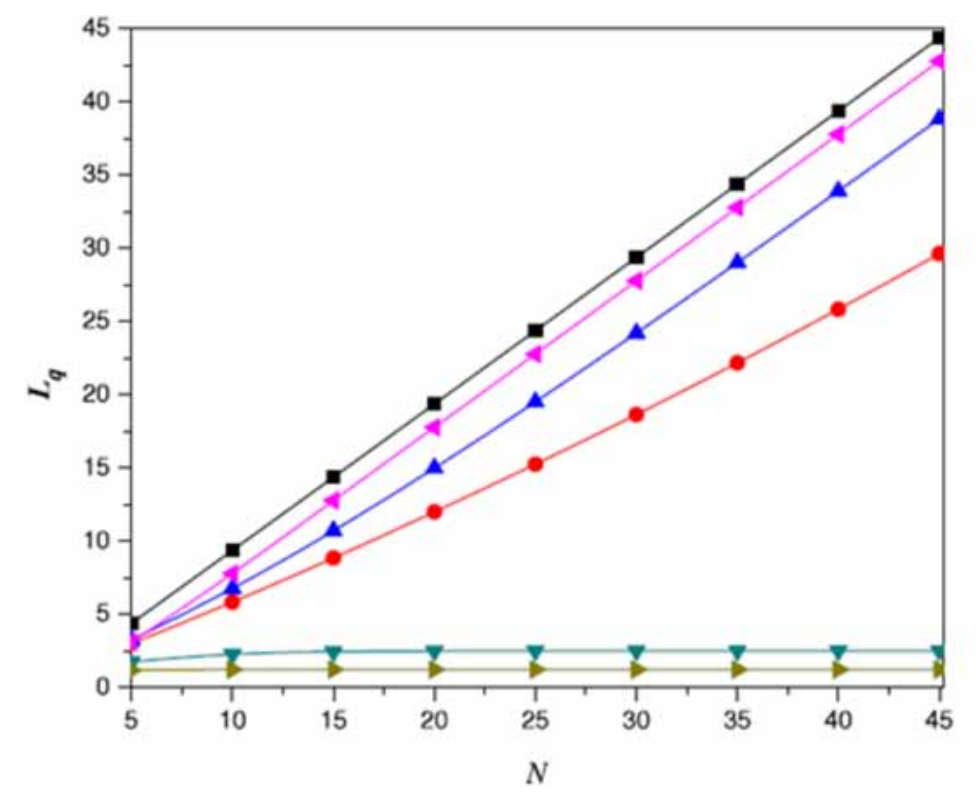

$+\lambda=14$

$\rightarrow \lambda=12$

$\star \lambda=10$

$+\lambda=8$

$+\lambda=6$

$+\lambda=4$

Figure 9. Effect of $\mathrm{N}$ on $\mathrm{W}$

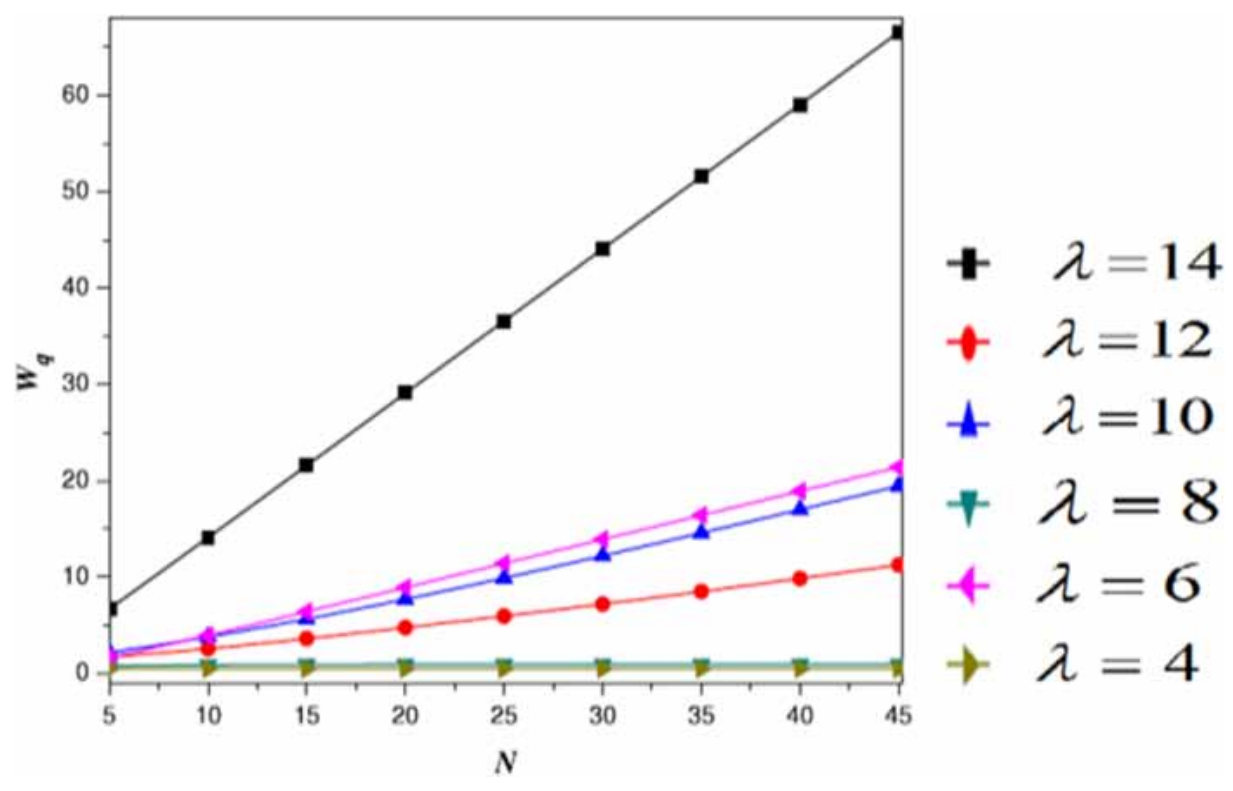


Figure 10. Effect on $\mathrm{N}$ on $\mathrm{Wq}$

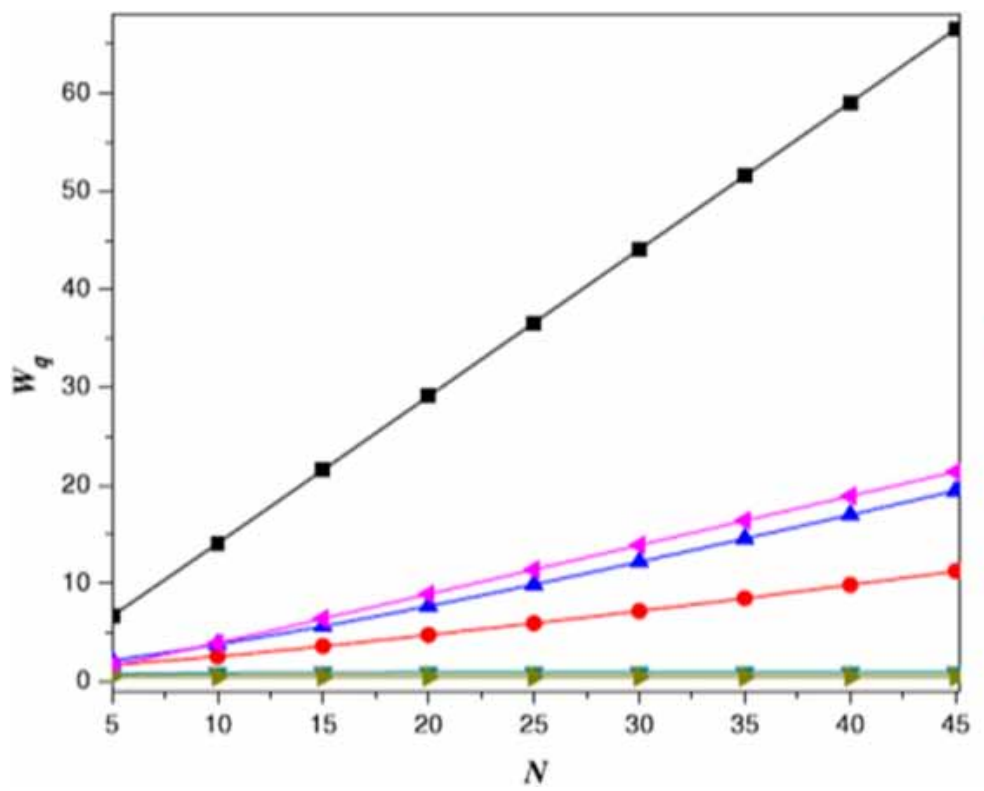

$+\lambda=14$

$+\lambda=12$

$₫ \lambda=10$

$+\lambda=8$

$+\lambda=6$

$\rightarrow \lambda=4$

Figure 11. Effect of blocking probability for different values of $b$ and $N$

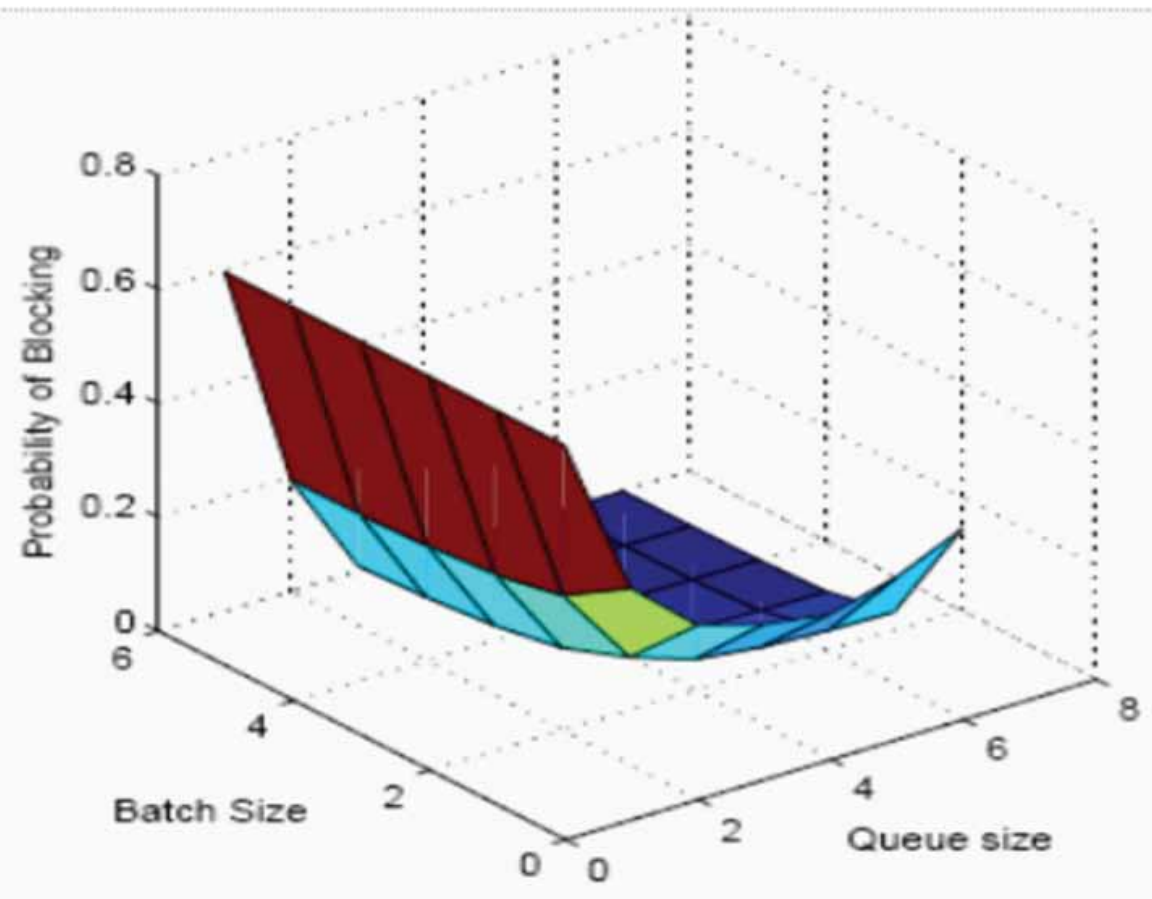




\section{CONCLUSION}

Blockchain and distributed ledger technology initially started with the cryptocurrencies and in the later stages, many research communities and the industries started evaluating and incorporating this technology into innumerable existing technologies. Blockchain Technology avidly facilitates several components of Smart City where Smart Healthcare plays a predominant role. In this particular scenario, a lot of patient data is constantly collected, recorded and analyzed by several healthcare professionals to provide efficient health services within the stipulated time. After several study it has been found that many mathematical models have also been built to prove the efficiency and authenticity of the application. Many uses cases have the same fundamental question of whether the use cases are going to give the required performance measures. To facilitate the decision, in this paper we have implemented $h Q C$ hain model i.e. a queueing model $\mathrm{M}^{1, \mathrm{~b}} / \mathrm{M}^{\mathrm{b}} / 1$ model that evaluates the various performance measures of the healthcare system and substantiates that such a model can prove to ensure an efficient Smart Healthcare System. The future research direction of this work include the studying the different real life parameters and their impact on different distributions. 


\section{REFERENCES}

Baker, S. B., Xiang, W., \& Atkinson, I. (2017). Internet of things for smart healthcare: Technologies, challenges, and opportunities. IEEE Access: Practical Innovations, Open Solutions, 5, 26521-26544. doi:10.1109/ ACCESS.2017.2775180

Barreiro-Gomez, J., \& Tembine, H. (2019). Blockchain token economics: A mean-field-type game perspective. IEEE Access: Practical Innovations, Open Solutions, 7, 64603-64613. doi:10.1109/ACCESS.2019.2917517

BK, B., \& Muralidhara, K. N. (2015). Secured smart healthcare monitoring system based on Iot. International Journal on Recent and Innovation Trends in Computing and Communication, 3(7), 4958-4961.

Carlozo, L. (2017). What is blockchain? Journal of Accountancy, 224(1), 29.

Catarinucci, L., De Donno, D., Mainetti, L., Palano, L., Patrono, L., Stefanizzi, M. L., \& Tarricone, L. (2015). An IoT-aware architecture for smart healthcare systems. IEEE Internet of Things Journal, 2(6), 515-526. doi:10.1109/JIOT.2015.2417684

Chiuchisan, I., Costin, H. N., \& Geman, O. (2014, October). Adopting the internet of things technologies in health care systems. In 2014 International Conference and Exposition on Electrical and Power Engineering (EPE) (pp. 532-535). IEEE. doi:10.1109/ICEPE.2014.6969965

Cruz, J. A., \& Wishart, D. S. (2006). Applications of machine learning in cancer prediction and prognosis. Cancer Informatics, 2. doi:10.1177/117693510600200030 PMID:19458758

da Conceição, A. F., da Silva, F. S. C., Rocha, V., Locoro, A., \& Barguil, J. M. (2018). Eletronic health records using blockchain technology. arXiv preprint arXiv:1804.10078.

Deakin, M., \& Al Waer, H. (2011). From intelligent to smart cities. Intelligent Buildings International, 3(3), 140-152. doi:10.1080/17508975.2011.586671

Dubovitskaya, A., Xu, Z., Ryu, S., Schumacher, M., \& Wang, F. (2017). Secure and trustable electronic medical records sharing using blockchain. AMIA ... Annual Symposium Proceedings - AMIA Symposium. AMIA Symposium, 2017, 650. PMID:29854130

Esteva, A., Kuprel, B., Novoa, R. A., Ko, J., Swetter, S. M., Blau, H. M., \& Thrun, S. (2017). Dermatologist-level classification of skin cancer with deep neural networks. Nature, 542(7639), 115-118. doi:10.1038/nature21056 PMID:28117445

Geissler, S., Prantl, T., Lange, S., Wamser, F., \& Hossfeld, T. (2019, August). Discrete-Time Analysis of the Blockchain Distributed Ledger Technology. In 2019 31st International Teletraffic Congress (ITC 31) (pp. 130137). IEEE. doi:10.1109/ITC31.2019.00029

Gupta, P., Agrawal, D., Chhabra, J., \& Dhir, P. K. (2016, March). IoT based smart healthcare kit. In 2016 International Conference on Computational Techniques in Information and Communication Technologies (ICCTICT) (pp. 237-242). IEEE. doi:10.1109/ICCTICT.2016.7514585

He, C., Fan, X., \& Li, Y. (2012). Toward ubiquitous healthcare services with a novel efficient cloud platform. IEEE Transactions on Biomedical Engineering, 60(1), 230-234. doi:10.1109/TBME.2012.2222404 PMID:23060318

He, D., Ye, R., Chan, S., Guizani, M., \& Xu, Y. (2018). Privacy in the Internet of Things for smart healthcare. IEEE Communications Magazine, 56(4), 38-44. doi:10.1109/MCOM.2018.1700809

Hillestad, R., Bigelow, J., Bower, A., Girosi, F., Meili, R., Scoville, R., \& Taylor, R. (2005). Can electronic medical record systems transform health care? Potential health benefits, savings, and costs. Health Affairs, 24(5), 1103-1117. doi:10.1377/hlthaff.24.5.1103 PMID:16162551

Islam, M. M., Rahaman, A., \& Islam, M. R. (2020). Development of Smart Healthcare Monitoring System in IoT Environment. SN Computer Science, 1(3).

Jayapandian, C. P., Chen, C. H., Bozorgi, A., Lhatoo, S. D., Zhang, G. Q., \& Sahoo, S. S. (2013). Cloudwave: Distributed processing of "Big Data" from electrophysiological recordings for epilepsy clinical research using Hadoop. AMIA ... Annual Symposium Proceedings - AMIA Symposium. AMIA Symposium, 2013, 691. PMID:24551370 
Joyia, G. J., Liaqat, R. M., Farooq, A., \& Rehman, S. (2017). Internet of Medical Things (IOMT): Applications, benefits and future challenges in healthcare domain. Journal of Communication, 12(4), 240-247.

Kasahara, S., \& Kawahara, J. (2016). Effect of Bitcoin fee on transaction-confirmation process. arXiv preprint arXiv:1604.00103.

Kawase, Y., \& Kasahara, S. (2017, August). Transaction-confirmation time for bitcoin: a queueing analytical approach to blockchain mechanism. In International Conference on Queueing Theory and Network Applications (pp. 75-88). Springer. doi:10.1007/978-3-319-68520-5_5

Kay, M., Santos, J., \& Takane, M. (2011). mHealth: New horizons for health through mobile technologies. World Health Organization, 64(7), 66-71.

Kemkarl, O. S., \& Dahikar, D. P. B. (2012). Can electronic medical record systems transform health care? potential health benefits, savings, and cost using latest advancements in ict for better interactive healthcare learning. International Journal of Computer Science \& Communication Networks, 2(3/6), 453-455.

Kiayias, A., Koutsoupias, E., Kyropoulou, M., \& Tselekounis, Y. (2016, July). Blockchain mining games. In Proceedings of the 2016 ACM Conference on Economics and Computation (pp. 365-382). ACM. doi:10.1145/2940716.2940773

Kiran, M. S., Rajalakshmi, P., Bharadwaj, K., \& Acharyya, A. (2014, March). Adaptive rule engine based IoT enabled remote health care data acquisition and smart transmission system. In 2014 IEEE World Forum on Internet of Things (WF-IoT) (pp. 253-258). IEEE. doi:10.1109/WF-IoT.2014.6803168

Kononenko, I. (2001). Machine learning for medical diagnosis: History, state of the art and perspective. Artificial Intelligence in Medicine, 23(1), 89-109. doi:10.1016/S0933-3657(01)00077-X PMID:11470218

Kumar, K. M., \& Venkatesan, R. S. (2014, May). A design approach to smart health monitoring using android mobile devices. In 2014 IEEE International Conference on advanced communications, control and computing technologies (pp. 1740-1744). IEEE. doi:10.1109/ICACCCT.2014.7019406

Kumar, T., Ramani, V., Ahmad, I., Braeken, A., Harjula, E., \& Ylianttila, M. (2018, September). Blockchain utilization in healthcare: Key requirements and challenges. In 2018 IEEE 20th International Conference on e-Health Networking, Applications and Services (Healthcom) (pp. 1-7). IEEE.

Lee, J. (2011, September). Smart health: concepts and status of ubiquitous health with smartphone. In ICTC 2011 (pp. 388-389). IEEE. doi:10.1109/ICTC.2011.6082623

Li, Q. L., Ma, J. Y., \& Chang, Y. X. (2018, December). Blockchain queue theory. In International Conference on Computational Social Networks (pp. 25-40). Springer.

Li, Q.-L., Ma, J.-Y., \& Chang, Y.-X. (2018). Blockchain Queue Theory. Springer Science and Business Media LLC. doi:10.1007/978-3-030-04648-4_3

Li, Q. L., Ma, J. Y., Chang, Y. X., Ma, F. Q., \& Yu, H. B. (2019). Markov Processes in Blockchain Systems. arXiv preprint arXiv:1904.03598.

Mettler, M. (2016, September). Blockchain technology in healthcare: The revolution starts here. In 2016 IEEE 18th international conference on e-health networking, applications and services (Healthcom) (pp. 1-3). IEEE.

Meyer, J., Heuten, W., \& Boll, S. (2016, September). No effects but useful? Long term use of smart health devices. In Proceedings of the 2016 ACM International Joint Conference on Pervasive and Ubiquitous Computing: Adjunct (pp. 516-521). doi:10.1145/2968219.2968314

Mukherjee, P., Barik, R. K., \& Pradhan, C. (2021). A Comprehensive Proposal for Blockchain-Oriented Smart City. In S. C. Tamane, N. Dey, \& A. E. Hassanien (Eds.), Security and Privacy Applications for Smart City Development. Studies in Systems, Decision and Control (Vol. 308). Springer. doi:10.1007/978-3-030-53149-2_4

Nakamoto, S. (2008). Bitcoin: A peer-to-peer electronic cash system. Academic Press.

Norman, C. D., \& Skinner, H. A. (2006). eHEALS: The eHealth literacy scale. Journal of Medical Internet Research, 8(4), e27. doi:10.2196/jmir.8.4.e27 PMID:17213046 
Obulor, R., \& Eke, B. O. (2016). Outpatient queueing model development for hospital appointment system. International Journal of Scientific Engineering and Applied Science, 2(4), 15-22.

Okada, S., Shiozawa, N., \& Makikawa, M. (2012, January). Body movement in children with adhd calculated using video images. In Proceedings of 2012 IEEE-EMBS International Conference on Biomedical and Health Informatics (pp. 60-61). IEEE. doi:10.1109/BHI.2012.6211505

Sahoo, S. S., Jayapandian, C., Garg, G., Kaffashi, F., Chung, S., Bozorgi, A., Chen, C.-H., Loparo, K., Lhatoo, S. D., \& Zhang, G. Q. (2013). Heart beats in the cloud: Distributed analysis of electrophysiological 'Big Data' using cloud computing for epilepsy clinical research. Journal of the American Medical Informatics Association: JAMIA, 21(2), 263-271. doi:10.1136/amiajnl-2013-002156 PMID:24326538

Solanas, A., Casino, F., Batista, E., \& Rallo, R. (2017, September). Trends and challenges in smart healthcare research: A journey from data to wisdom. In 2017 IEEE 3rd International Forum on Research and Technologies for Society and Industry (RTSI) (pp. 1-6). IEEE. doi:10.1109/RTSI.2017.8065986

Sukumar, S. R., Natarajan, R., \& Ferrell, R. K. (2015). Quality of Big Data in health care. International Journal of Health Care Quality Assurance, 28(6), 621-634. doi:10.1108/IJHCQA-07-2014-0080 PMID:26156435

Syed, L., Jabeen, S., Manimala, S., \& Elsayed, H. A. (2019). Data science algorithms and techniques for smart healthcare using IoT and big data analytics. In Smart Techniques for a Smarter Planet (pp. 211-241). Springer. doi:10.1007/978-3-030-03131-2_11

Yang, Z., Zhou, Q., Lei, L., Zheng, K., \& Xiang, W. (2016). An IoT-cloud based wearable ECG monitoring system for smart healthcare. Journal of Medical Systems, 40(12), 286. doi:10.1007/s10916-016-0644-9 PMID:27796840

Yin, H., Akmandor, A. O., Mosenia, A., \& Jha, N. K. (2018). Smart healthcare. Academic Press.

Zeadally, S., Siddiqui, F., Baig, Z., \& Ibrahim, A. (2019). Smart healthcare: Challenges and potential solutions using internet of things (IoT) and big data analytics. PSU research review.

Zhu, H., Wu, C. K., Koo, C. H., Tsang, Y. T., Liu, Y., Chi, H. R., \& Tsang, K. F. (2019). Smart Healthcare in the Era of Internet-of-Things. IEEE Consumer Electronics Magazine, 8(5), 26-30. doi:10.1109/MCE.2019.2923929

Zhuang, Y., Sheets, L. R., Chen, Y. W., Shae, Z. Y., Tsai, J. J., \& Shyu, C. R. (2020). A patient-centric health information exchange framework using blockchain technology. IEEE Journal of Biomedical and Health Informatics, 24(8), 2169-2176. doi:10.1109/JBHI.2020.2993072 PMID:32396110

Pratyusa Mukherjee is a PhD Scholar of School of Computer Engineering, KIIT Deemed to be University, Bhubaneshwar, India. She received a B. Tech in Electronics and Communication Engineering from West Bengal University of Technology and $M$. Tech in Information Technology from Indian Institute of Engineering Science and Technology, Shibpur. Her research area is in the field of Information Security. It includes Chaos based Encryption, lightweight block cipher, Blockchain Technology and DNA Cryptography.

Chittaranjan Pradhan has obtained his Doctorate, Masters and Bachelor degrees in Computer Science \& Engineering discipline. Currently, he is working as Associate Professor at School of Computer Engineering, Kalinga Institute of Industrial Technology (KIIT) Deemed to be University, Bhubaneswar, India. Dr. Pradhan has got a total of 14 years of Academic Teaching experience with more than 70 publications in reputed \& peer reviewed Journals, Edited Books and Conferences of National and International repute. His research area includes Information Security, Image Processing, Deep Learning, Multimedia Systems. He has published few books published by publishers like LAP Lambert, IGI Global, Elsevier. He is also member of various National and International Professional Societies in the field of Engineering \& Research such as: IET, IACSIT, CSI, ISCA, IAENG, ISTE.

Sudhansu Shekhar Patra is currently an Associate Professor in the School of Computer Application, KIIT University, Bhubaneswar, India. He received his Master degree in Computer Application from Motilal Nehru National Institute of Technology, Allahabad, India, M. Tech(Computer Science \& Engg) from Utkal University, Bhubaneswar, India and Ph.D. in Computer Science from KIIT University, Bhubaneswar, India. His research interests include grid computing, Cloud Computing, Machine Learning, SDN, Algorithms. He is a life member of Indian Society for Technical Education.

Rabindra K. Barik is currently working as an Assistant Professor in the School of Computer Applications, Kalinga Institute of Industrial Technology, Bhubaneswar, India. He has received his both M. Tech and Ph.D. in Geoinformatics from Motilal Nehru National Institute of Technology, Allahabad, India. His research area includes Geospatial Database, SOA, Cloud Computing, Fog computing, IPR and Geoinformatics. He is a member of IEEE and IAENG. 